Short Communication

Human and Medical Genetics

\title{
First description of ultramutated endometrial cancer caused by germline loss-of-function and somatic exonuclease domain mutations in POLE gene
}

\author{
Reginaldo Cruz Alves Rosa ${ }^{1 *}$ (iD, Andrey A. Yurchenko ${ }^{2 *}$, Fernando Chahud ${ }^{3}$, Alfredo Ribeiro-Silva ${ }^{3}$, \\ Mariângela Ottoboni Brunaldi ${ }^{3}$, Wilson Araújo Silva $\mathrm{Jr}^{1}$ iD, Patricia L. Kannouche ${ }^{4}$, Sergey Nikolaev², \\ Victor Evangelista de Faria Ferraz ${ }^{1,5}$ (iD \\ ${ }^{1}$ Universidade de São Paulo, Faculdade de Medicina de Ribeirão Preto, Departamento de Genética, \\ Ribeirão Preto, SP, Brazil. \\ ${ }^{2}$ Université Paris Saclay, Inserm U981, Gustave Roussy Cancer Campus, Villejuif, France. \\ ${ }^{3}$ Universidade de São Paulo, Faculdade de Medicina de Ribeirão Preto, Departamento de Patologia e \\ Medicina Legal, Ribeirão Preto, SP, Brazil. \\ ${ }^{4}$ Paris-Sud University, CNRS-UMR 8200, Equipe labellisée Ligue Contre le Cancer, Gustave Roussy Cancer \\ Campus, Villejuif, France. \\ ${ }^{5}$ Universidade de São Paulo, Centro de Genômica Médica, Hospital das Clínicas, Faculdade de Medicina de \\ Ribeirão Preto, Ribeirão Preto, SP, Brazil.
}

\begin{abstract}
Endometrial cancer (EC) harboring heterozygous POLE proofreading inactivating mutations (POLE-exo*) is associated with an increased number of somatic mutations that result in a distinctive anti-tumor immune response. However, the consequences of such POLE mutations in the context of the missing wild-type allele have not yet been described in endometrial tumors. A 72-year-old woman harboring a germline monoallelic frameshift mutation (p.Pro269fsTer26) in POLE was diagnosed with an EC having a somatic heterozygous mutation in the exonuclease domain of $P O L E$ (S459F). Targeted gene sequencing revealed an ultramutated phenotype (381 mutations/Mb) in the tumor and a 2-fold excess of mutations on the DNA leading strand. Additionally, we observed a mutational signature similar to the COSMIC signature 10, a higher mutation rate in this tumor than in endometrial tumors with heterozygous POLE-exo*, and an increased number of $\mathrm{T}$ lymphocytes. This is the first report of an ultramutated EC harboring a somatic POLE-exo* mutation in association with a germline loss-of-function mutation in this gene. The absence of a wild type $P O L E$ allele led to a particularly high mutational burden.
\end{abstract}

Keywords: Endometrial cancer, POLE exonuclease mutation, Targeted sequencing, TMB, Ultramutated phenotype.

Received: April 9, 2020; Accepted: July 8, 2020.

Endometrial cancer (EC) is a heterogeneous malignancy characterized by several different histologic subtypes with endometrioid carcinoma being the most common (McConechy et al., 2016). Recently, there have been significant advances in defining the molecular alterations that contribute to tumorigenesis in EC. The Cancer Genome Atlas Research Network (TCGA) divides EC into four categories based on recurrent molecular features: an ultramutated phenotype caused by POLE mutations, a hypermutator phenotype caused by the DNA mismatch repair deficiency (MMRD) leading to microsatellite instability (MSI), a copy number low phenotype, and a copy number high phenotype (Levine et al., 2013).

Send correspondence to Victor Evangelista de Faria Ferraz. Universidade de São Paulo, Faculdade de Medicina de Ribeirão Preto, Departamento de Genética, Av. Bandeirantes, 3900, Vila Monte Alegre, 14049-900, Ribeirão Preto, SP, Brazil. E-mail: vferraz@usp.br.

* These authors have contributed equally to this work.
The POLE gene encodes the catalytic subunit of DNA polymerase $\varepsilon(\mathrm{Pol} \varepsilon)$, which replicates the leading strand during DNA replication (Burgers et al., 2017). In addition to DNA-binding and polymerase domains, Pol $\varepsilon$ has proofreading activity through its exonuclease domain. This capacity is essential for the maintenance of replication fidelity, and this proofreading function may act, not only on newly misincorporated nucleotides, but also on mismatches produced by non-proofreading polymerases (Palles et al., 2013). Up to $12 \%$ of all endometrial carcinomas harbor POLE mutations that tend to cluster in the exonuclease domain (POLE-exo*), especially in the conserved residues 268 to 471 (Billingsley et al., 2016; Bellone et al., 2017; Barbari et al., 2018). Tumors harboring such mutations are associated with an ultramutated phenotype, increased neoantigen load, increased tumor infiltrating lymphocytes, and increased potential for responding to immunotherapy (Imboden et al., 2019). 
Germline mutations in the exonuclease domain of $P O L E$ are infrequent; most POLE-exo* mutations are somatic and occur in sporadic tumors almost exclusively in a heterozygous state because their dominant nature (Wong et al., 2016; Barbari et al., 2017). Additionally, there is no associated POLE inactivation by somatic loss of heterozygosity $(\mathrm{LOH})$ when tumors occur in carriers of germline POLE mutations (Palles et al., 2013). However, a minority of tumors with $P O L E$-exo* show LOH or other inactivating mutations that could act as 'second hits' (Heitzer et al., 2014). Curiously, loss or inactivation of the second allele has been reported in a few colorectal tumors with mutations disturbing Pol $\varepsilon$ proofreading activity and at least one example illustrates that this mutation may have phenotypic consequences for disease presentation (Muzny et al., 2012). However, similar findings have not been reported for endometrial tumors (Shinbrot et al., 2014).

Here, we report a 72-year-old woman diagnosed with a FIGO Grade 1 and FIGO Stage 1B endometrial endometrioid adenocarcinoma at 63 years old. A total hysterectomy and salpingo-oophorectomy were performed. The patient reported no familial history of cancer. Immunohistochemistry (IHC) of the MMR proteins and MSI analysis were performed. The tumor had an intact expression of MLH1, MSH2, MSH6 and PMS2 proteins, based on immunohistochemical analysis, and was classified as MSI-low based on the MSI assay. A germline and somatic mutation screening were performed, and the mutational profile and its immunologic characterization of the endometrial tumor were accessed (for details of material and methods, see Mat-Met S1 in Supplementary Material). The study was approved by the Scientific and Research Committee of the Clinics Hospital of the Ribeirão Preto Medical School (protocol number: 1.578.206). Informed written consent was obtained from the patient.

For germline mutation screening, a targeted sequencing assay of the coding, canonical splice sites, and both 5' and 3' untranslated regions of 63 genes (Table S1), including Lynch syndrome-associated genes and POLE, was performed in DNA extracted from peripheral blood. Single nucleotide variants (SNVs) and Copy number variation (CNV) were evaluated. Only the germline frameshift mutation NM_006231:c.806delC (p.Pro269fsTer26) in POLE was identified (Figure 1A), with a variant allele frequency (VAF) of 0.50 (total coverage $=729$ reads), as expected for a heterozygous germline variant.

Since this germline mutation could not explain the tumor MSI-low phenotype present in the EC, a further mutational search was performed using the tumor DNA. For somatic analysis, targeted sequencing using the same gene panel described for germline analysis was performed on genomic DNA extracted from a representative tumor area (at least $70 \%$ of tumor cells) from Formalin-Fixed ParaffinEmbedded (FFPE) blocks. Both somatic SNVs and CNVs were called on the matching tumor-blood DNA samples. As expected, the germline frameshift mutation in $P O L E$ was also detected in tumor sequencing, with a $\mathrm{VAF}=0.51$ (total coverage $=242$ reads , supporting its germline origin. Additionally, a somatic mutation in the exonuclease domain of POLE, S459F (NM 006231:c.1376C > T, p.Ser459Phe), was observed with $\mathrm{VAF}=0.298$ (Figure 1B). As long as this mutation is heterozygous, it is expected to be present in $\sim 60 \%$ of cells in the tumor sample, these estimates are based on a tumor purity of $80 \%$ from the histological examination. We did not find any pathogenic mutation neither in the MMR genes (MLH1, MSH2, MSH6, and PMS2) not in the exonuclease domain of POLD1.

The tumor mutational profile was investigated to determine whether the genomic alterations were consistent with a POLE ultramutator phenotype. A total of 190 mutations were identified in the sequenced region of the 63 gene panel (0.49 Mb). Considering only the coding region, 0.257 $\mathrm{Mb}$ distributed along 937 exons of 63 cancer-related genes, a total of 95 mutations were identified, resulting in a mutation rate of 381 mutations/Mb. A total of 65 nonsynonymous mutations were identified in the targeted exons, resulting in an estimated tumor mutation burden (TMB) of 253 nonsynonymous mutations/Mb.

The trinucleotide context of mutations was investigated, and a mutational signature analysis was performed using the database of the known mutational signatures in human cancers from Alexandrov et al. (2013). Given the high number of somatic mutations identified, we had sufficient data to derive a mutational signature that was closely related to COSMIC signature 10 (Cosine similarity $=0.97$, Figure 2A). These findings are indicative of mutations in DNA replication associated with errors in proofreading activity of Pol $\varepsilon$. Most nucleotide substitutions detected in the tumor sample were represented by $\mathrm{C}>\mathrm{A}, \mathrm{C}>\mathrm{T}$, and $\mathrm{T}>\mathrm{G}$, with a relative contribution to the total amount of substitution mutations of $0.43,0.33$ and 0.18 , respectively (Figure 2B).

In addition to mutational signature analysis, we calculated the mutation strand bias asymmetry between the leading and lagging DNA strands. There was a 2-fold excess of mutations on the leading strand of DNA in comparison with the lagging strand (Figure 2C). These molecular findings highlight the strong effect of defects in Pol $\varepsilon$ proofreading activity in this reported EC.

In order to compare the mutation rate and total TMB between the studied tumor and endometrial cancers with heterozygous $P O L E$-exo* mutations, we downloaded 25 exomes of endometrial carcinoma from ICGC portal with POLE-exo* somatic mutations and absence of MSI (Zhang et al., 2011). To minimize the influence of different capture kits we estimated TMB only in the coding regions of our gene panel. The mutation rate observed in the endometrial carcinoma reported here (381 mutations/Mb) is more than 2-fold higher than the average rate observed in 25 endometrial carcinomas harboring heterozygous POLE-exo* mutations (153 mutations/Mb, ranging from 47 to 276 ). One out of 25 endometrial carcinomas harbored the heterozygous POLE-exo* S459F and presented a rate of 167 mutations/Mb (Table 1). These data confirm that EC harboring a 
A

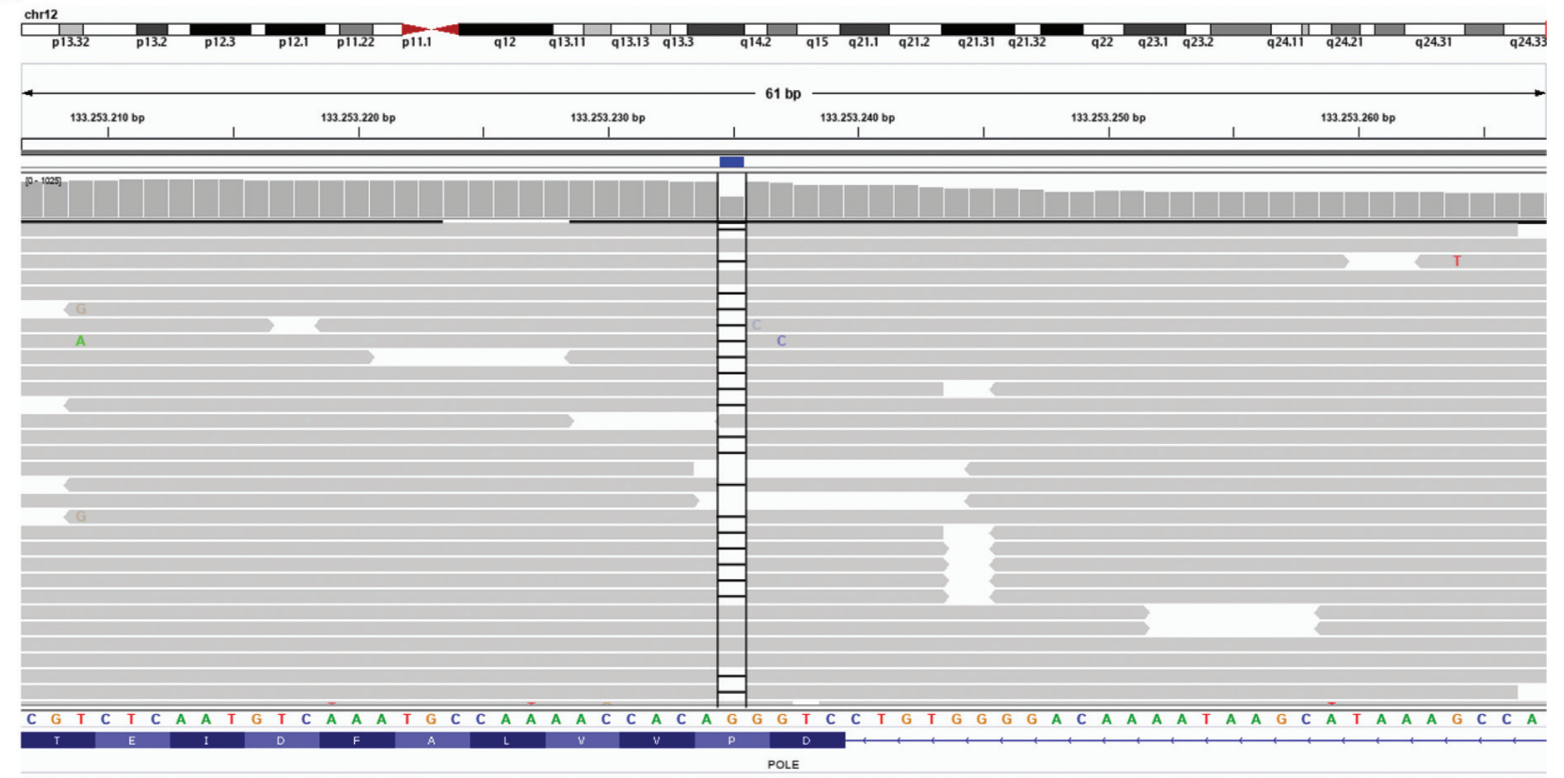

B
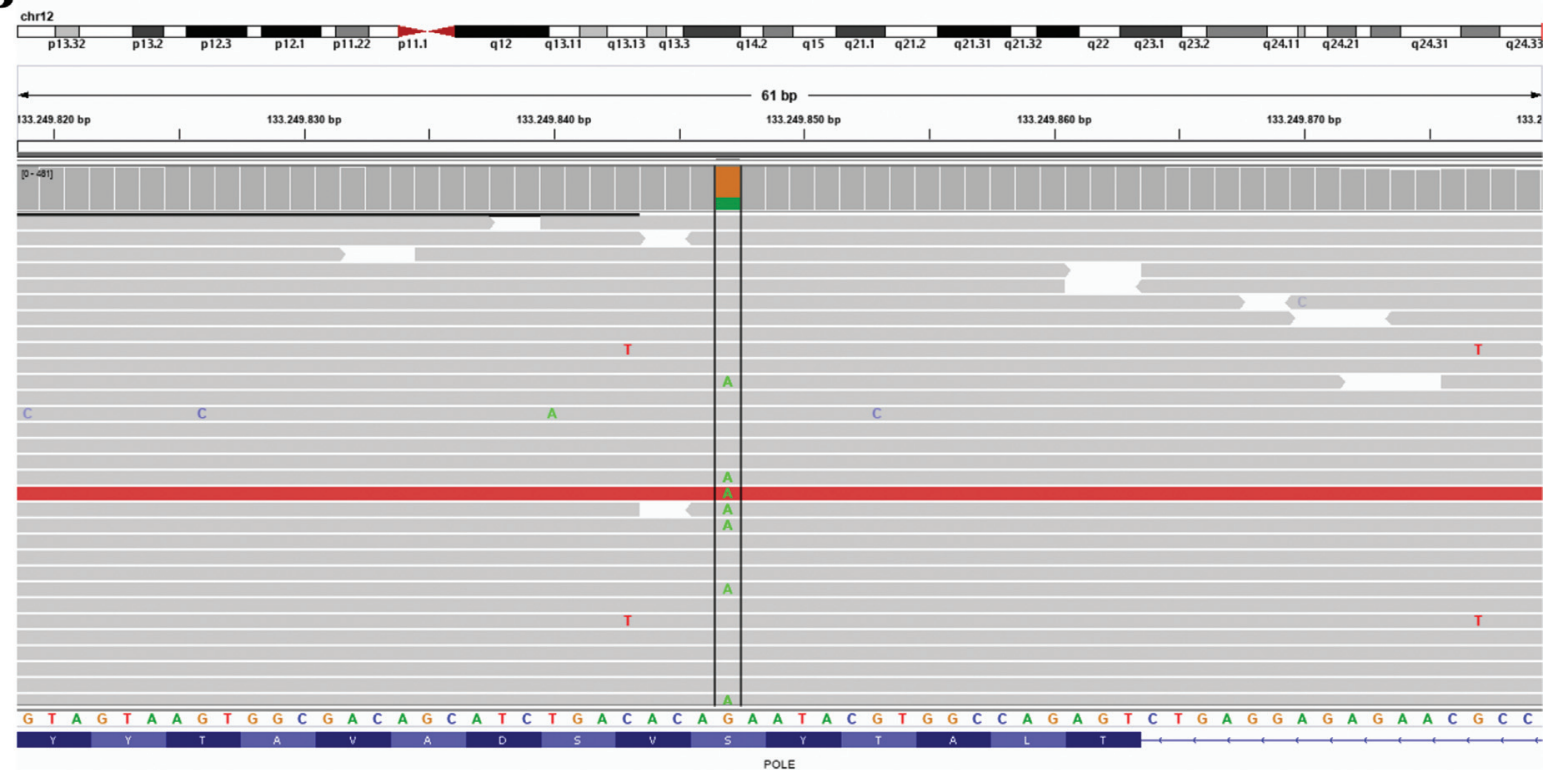

POLE

Figure 1 - Integrative Genomics Viewer snapshot of POLE mutations with reference POLE nucleotide and amino acid sequences. (A) germline frameshift c.806delC and (B) somatic c.1376C $>$ T (S459F) exonuclease mutation.

LoF genetic event in POLE in association with a heterozygous $P O L E$-exo* mutation confers an augmented mutator phenotype in comparison with EC with single POLE-exo* alterations.

For evaluation of tumor-associated lymphocytes, the mean number of $\mathrm{CD} 3+, \mathrm{CD} 4+$, and $\mathrm{CD} 8+$ of intraepithelial $\mathrm{T}$ lymphocytes, i.e., $\mathrm{T}$ lymphocytes located within the tumor epithelium was calculated. IHC staining for T lymphocyte markers revealed a predominance of $\mathrm{CD} 8+$ lymphocytes in the intra-tumoral area in comparison with $\mathrm{CD} 4+\mathrm{T}$ cells, with mean numbers of $29.9 \mathrm{CD} 8+$, and $10.9 \mathrm{CD} 4+\mathrm{T}$-cells. A mild (1+) presence of CD3+, CD4+, and CD8+ lymphocytes was observed in the peri-tumoral region (Figure 3).
This is the first case of an endometrial carcinoma harboring a somatic POLE exonuclease mutation related to an ultra-mutator phenotype acting as a 'second hit' in association with a germline truncating mutation of the gene. Germline heterozygous missense mutations affecting the POLE exonuclease domain are associated with a syndrome called Polymerase Proofreading-Associated Polyposis (PPAP) that increases the risk for the development of multiple colorectal adenomas and colorectal cancer (Briggs et al., 2013). A diagnosis of PPAP is not consistent with findings in the patient presented in this case report since she carries a germline frameshift mutation at the beginning of the POLE exonuclease domain that creates a premature termination 

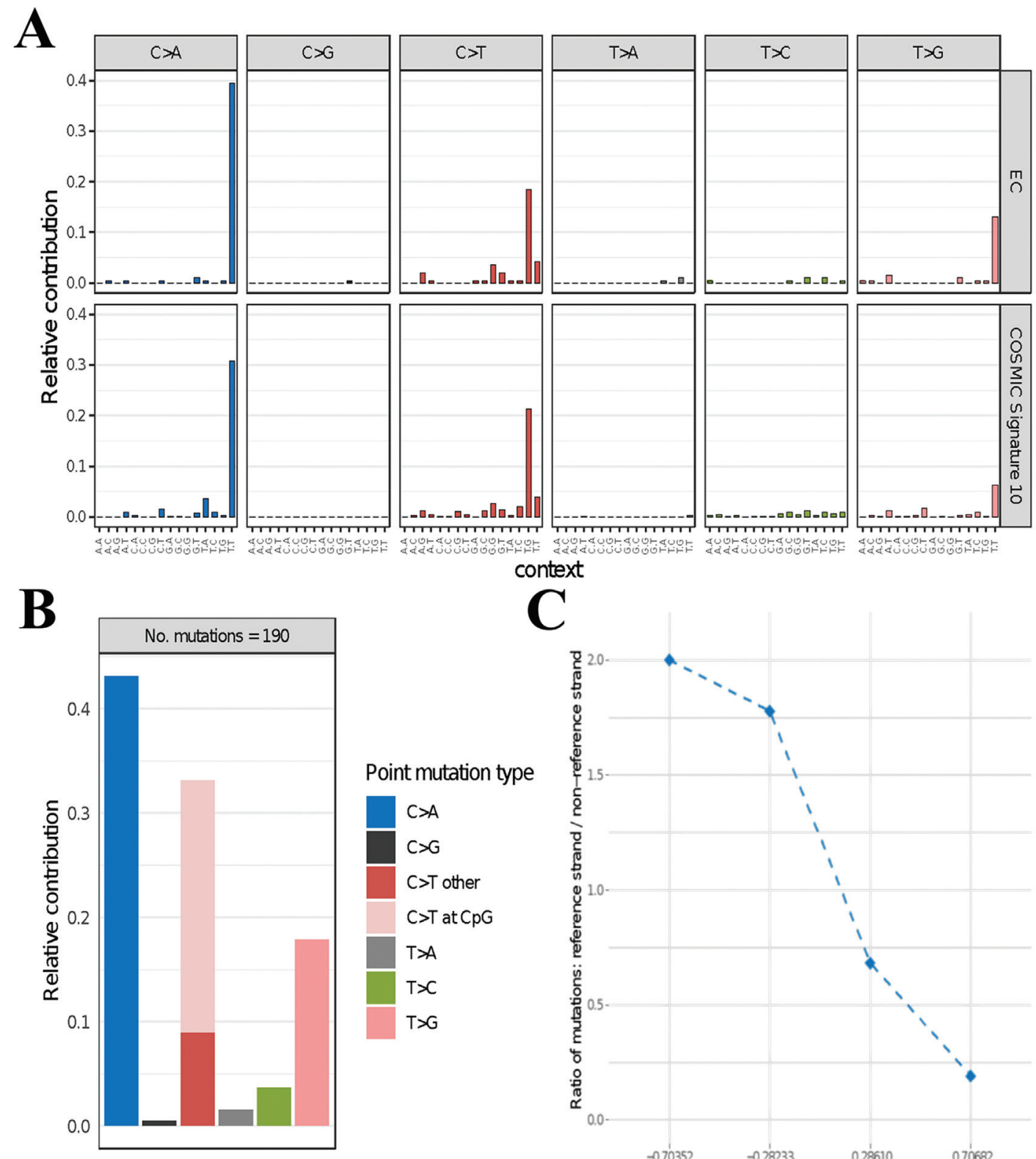

context

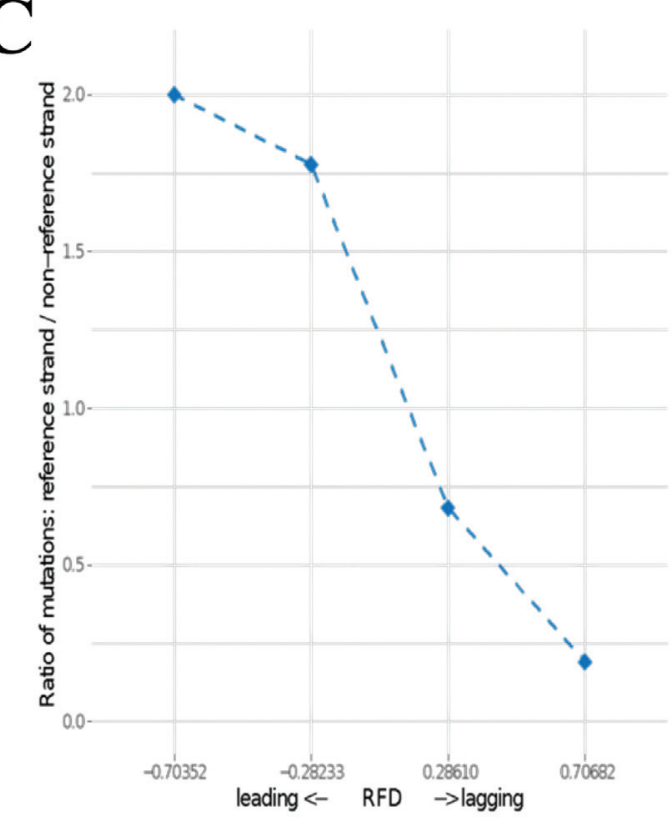

Figure 2 - Mutational profile based on targeted sequencing data of a 63 cancer-related gene panel. (A) mutational signature extracted from the endometrial carcinoma in comparison to the COSMIC signature 10. (B) relative contribution of each point mutation type to the total amount of somatic mutations. (C) Strand bias analysis showing a predominance of mutations on the leading strand. EC, endometrial cancer.

codon. Truncating mutations in POLE gene are unlikely to lead to PPAP phenotype, since a successful DNA synthesis must occur before the proofreading activity of Pol $\varepsilon$ (Heitzer et al., 2014). However, the co-occurrence of a germline truncating mutation with a somatic ultra-mutator phenotype-associated variant in POLE suggests a complete loss of Pol $\varepsilon$ proofreading activity in the endometrial tumor. Thus, by itself the germline frameshift mutation does not confer a genetic predisposition to EC and cannot lead to a mutator phenotype in the tumor, but might contribute to increase the mutational load because only proofreading-deficient Pol- $\varepsilon$ will replicate DNA in these tumor cells.
Some POLE-exo* mutations have been described to be associated with an ultra-mutator phenotype, with varying levels of mutation. Previous functional studies have demonstrated the exonuclease deficiency effect of the POLE S459F mutation in vitro (Shinbrot et al., 2014) as well as the moderate mutator effect in yeast (Barbari et al., 2018). The EC reported here was MSI-low. Co-occurrence of MSI and POLE-exo* mutations, usually with the P286R mutation, in endometrial tumors has already been reported (Haradhvala et al., 2018). However, all tumors described to date that harbor the $\mathrm{S} 459 \mathrm{~F}$ mutation in POLE were found to be microsatellite stable (Shinbrot et al., 2014; Andrianova et al., 2017; Barbari et al., 2018). Our study is the first report of a 
Table 1 - Mutational profile of endometrial carcinomas harboring heterozygous $P O L E$-exo* mutations in comparison with the endometrial cancer reported.

\begin{tabular}{|c|c|c|c|c|c|}
\hline Sample & $\begin{array}{c}P O L E \text {-exo* } \\
\text { mutation }\end{array}$ & $\begin{array}{l}\text { Total amount } \\
\text { of mutations }\end{array}$ & $\begin{array}{c}\text { Muta- } \\
\text { tions/ } \\
\text { Mb }\end{array}$ & $\begin{array}{c}\text { Nonsynony- } \\
\text { mous } \\
\text { mutations }\end{array}$ & $\mathrm{TMB}^{\mathrm{b}}$ \\
\hline Report & $\mathrm{S} 459 \mathrm{~F}$ & 95 & 381 & 65 & 253 \\
\hline SA485042 & P286R & 71 & 276 & 53 & 206 \\
\hline SA475378 & P286R & 70 & 272 & 47 & 183 \\
\hline SA552345 & P286R & 69 & 268 & 44 & 171 \\
\hline SA472709 & P286R & 59 & 230 & 42 & 163 \\
\hline SA466958 & P286R & 61 & 237 & 41 & 160 \\
\hline SA470974 & P286R & 64 & 249 & 41 & 160 \\
\hline SA462048 & P286R & 63 & 245 & 39 & 152 \\
\hline SA467568 & P286R & 47 & 183 & 35 & 136 \\
\hline SA483959 & P286R & 48 & 187 & 32 & 125 \\
\hline SA541518 & P286R & 39 & 152 & 29 & 113 \\
\hline SA472897 & S459F & 43 & 167 & 29 & 113 \\
\hline SA20267 & P286R & 38 & 148 & 27 & 105 \\
\hline SA561528 & P286R & 49 & 191 & 24 & 93 \\
\hline SA469202 & P286R & 31 & 121 & 20 & 78 \\
\hline SA473549 & P286R & 26 & 101 & 20 & 78 \\
\hline SA526095 & P286R & 28 & 109 & 18 & 70 \\
\hline SA541610 & P286R & 24 & 93 & 18 & 70 \\
\hline SA526120 & P286R & 26 & 101 & 15 & 58 \\
\hline SA92158 & P286R & 29 & 113 & 15 & 58 \\
\hline SA482148 & P286R & 24 & 93 & 13 & 51 \\
\hline SA479614 & P286R & 19 & 74 & 12 & 47 \\
\hline SA467039 & V411L & 16 & 62 & 10 & 39 \\
\hline SA476079 & V411L & 15 & 58 & 9 & 35 \\
\hline SA474561 & P286R & 12 & 47 & 7 & 27 \\
\hline SA519177 & P286R & 12 & 47 & 6 & 23 \\
\hline
\end{tabular}

a all mutations were identified along the $0.257 \mathrm{Mb}$ of the 63 gene panel, including synonymous and nonsynonymous mutations. ${ }^{\mathrm{b}}$ expressed as number of nonsynonymous mutations/Mb. TMB, tumor mutational burden. Report: endometrial cancer case characterized in the present study.

tumor harboring the S459F mutation this is associated with an MSI-low phenotype.

Somatic mutations found in cancer genomes are the consequence of the intrinsic infidelity of the DNA replication machinery, exogenous or endogenous mutagen exposures, enzymatic modification of DNA, or defective DNA repair and other processes. Different mutational processes often generate variation in the combinations of mutation types, termed mutational signatures (Alexandrov et al., 2013). More than 30 mutational signatures have already been identified across 40 different types of human cancer (Forbes et al., 2017). We identified a mutational signature that closely resembles the COSMIC signature 10 , which is known to be associated with POLE-exo* mutations (Alexandrov et al., 2013). The POLE mutational signature is characterized by a 100 -fold increase in $\mathrm{C}>\mathrm{A}$ transversions in the context TCT and a 30-fold increase in $\mathrm{C}>\mathrm{T}$ transitions in the context TCG (Rayner et al., 2016). This mutational pattern results in a strong bias for particular amino acid changes, with an overrepresentation of serine to tyrosine or leucine, and arginine to isoleucine or glutamine substitutions, and a substantial increase in glutamic acid to stop codon mutations (Rayner et al., 2016). Although mutational signatures are preferably determined by genomic analysis, such as whole genome sequencing (WGS) and whole exome sequencing (WES), we were able to identify a mutational signature related to $P O L E$-exo* mutations through targeted sequencing of the coding and regulatory regions of only 63 genes. These findings support that mutational signatures can be extracted from sequencing data derived from a small gene panel in tumors that are highly mutated (Hoeck et al., 2019). In addition, we observed a strong strand bias effect with mutations occurring predominantly in the leading strand in comparison with the lagging strand. This phenomenon, in addition to the mutational signature close to COSMIC signature 10, highlights the major effect of POLE proofreading inactivation in the $\mathrm{EC}$ reported here.

TMB is a quantitative measure of the total number of somatic nonsynonymous mutations per coding area of a tumor genome and is associated with the emergence of neoantigens that trigger anti-tumor immunity (Allgäuer et al., 2018; Meléndez et al., 2018). We identified a total of 65 nonsynonymous mutations along $0.257 \mathrm{Mb}$ coding regions of the sequenced gene panel, resulting in an estimated TMB of 253 nonsynonymous mutations/ $\mathrm{Mb}$. Although a wider genomic analysis is required to achieve the precise TMB (Büttner et al., 2019), the absolute amount of somatic nonsynonymous mutations (65 mutations/ $0.257 \mathrm{Mbp}$ ) observed in the EC reported here is superior to the threshold of $20 \mathrm{mu}-$ tations $/ \mathrm{Mb}$ commonly used to classify a tumor with high TMB and as an immunotherapy responder (Allgäuer et al., 2018; Endris et al., 2019). The absolute amount of nonsynonymous mutations, as well as the total number of mutations (95 mutations/0.257) identified in our report is higher than those identified in EC harboring heterozygous POLEexo* mutations. We used the GATK pipeline, which has a high sensitivity and specificity for somatic mutations calling and checked the occurrence of FFPE-derived artefacts in the sequencing data. Thus, the higher mutational load identified in the endometrial tumor in comparison with the ICGC/TCGA Pole-exo* tumors is not supposed to be led by interstudy differences.

The occurrence of two proofreading-inactivating events in POLE is extremely rare, suggesting that POLE may not act as a classical tumor suppressor gene (Heitzer et $a l ., 2014)$. There is a single case of colorectal cancer (CRC) in The Cancer Genome Atlas (TCGA) project carrying the $P O L E$ S459F mutation and a nonsense mutation at codon 150 of the POLE gene, which was thought to inactivate the second allele (Muzny et al., 2012). This CRC presented a higher number of somatic mutations (376 mutations/ $\mathrm{Mb}$ ) in comparison with another TCGA-CRC harboring only the S459F mutation in heterozygosity (81 mutations/Mb) (Shin- 

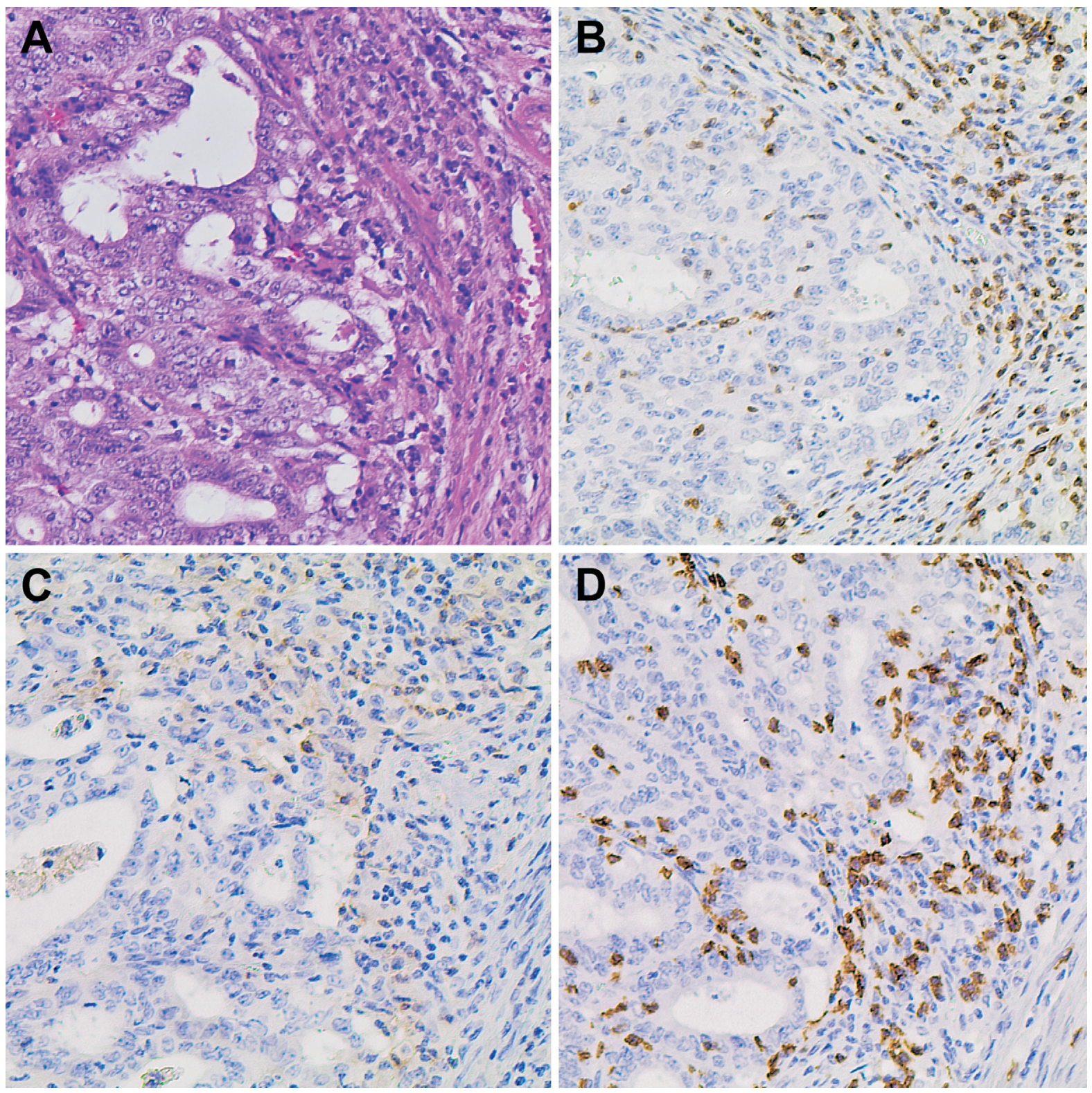

Figure 3 - Immunohistochemical staining for T lymphocyte markers on the peri and intra-tumoral areas of the EC (original magnification x200) . (A) $\mathrm{H} \& \mathrm{E}$ (hematoxilin-eosin). Brown nuclear staining is indicative of positive expression (B) CD3, (C) CD4 and (D) CD8 markers.

brot et al., 2014). Both mutations present in the TCGA-CRC with two hits in POLE are somatic. Our findings are novel since we report an endometrial carcinoma harboring one germline POLE LoF mutation and one somatic POLE-exo* mutation.

Molecular classification of human cancer represents an important step toward the goal of precision medicine and helps to identify patients who would benefit from targeted immunotherapy (Liu et al., 2019). We observed the occurrence of a greater number of CD8+ T lymphocytes in comparison with $\mathrm{CD} 4+\mathrm{T}$-cells in the peri and intra-tumoral area in our EC case. POLE-exo* mutations have been associated with increased tumor infiltrating lymphocytes, especially CD8+ (Howitt et al., 2015; Bourdais et al., 2017).
The characterization of the mutational pattern, as well as the lymphocyte profile revealed an accentuated Pol $\varepsilon$ proof-reading failure in an EC harboring a germline and a somatic mutation at the POLE exonuclease domain. These findings suggest that the mutations are in trans, i.e. located in different DNA strands. The frameshift mutation affects the beginning of the exonuclease domain of POLE and is expected to result in a truncated, immature, or non-functional protein. If the POLE S459F mutation were located at the same strand as the germline frameshift, the ultramutator effect would likely be silenced by the frameshift. However, as a limitation of our study, we could not experimentally prove that the frameshift and missense POLE-exo* mutations are in trans and neither that the frameshift indeed led to the silencing of one POLE allele, due to the high fragmentation of 
DNA and RNA derived from FFPE slides. Additionally, although we have strong evidence supporting that our EC case has a higher mutational load identified in comparison with tumors harboring heterozygous POLE-exo* mutations, we are aware that the number of mutations identified might have been affected by interstudy differences in sample preservation methods, library protocols, and bioinformatic pipelines. Also, although we observed a higher mutational load in the EC with two genetic events at the proof-reading domains of $P O L E$ in comparison with EC harboring only a heterozygous POLE-exo* mutation, we would need to have more tumors with similar findings in order to make statistically significant conclusions about the mutational burden of these tumors relative to the cancers with heterozygous POLE-exo* mutations.

In conclusion, our EC case exhibits molecular and histopathological features typically linked to POLE exonuclease mutated tumors. The comparison with other tumors with $P O L E$-exo* mutations suggests that the absence of the wild type $P O L E$ allele renders particularly higher TMB in such tumors. Consequently, detection of a combination of POLE-exo* and LoF POLE mutations could be considered as prognostic or therapeutic marker.

\section{Acknowledgments}

The authors would like to thank the patient for her kind collaboration and contribution to this publication. Also, we thank Dr. Jeremy Squire for his technical support during the manuscript review. The costs of the design of the study and molecular analyses were supported by FAPESP (grant 2017/04283-8); CNPq (grant 423007/2016-5?). Analysis and interpretation of data were supported by Foundation ARC 2017, Foundation Gustave Roussy and Swiss Cancer League (grant KFC-3985-08-2016), and INCa (INCaPLBio2016-144 and INCa-DGOS-Inserm 12551).

\section{Conflict of interest}

The authors declare that there is no conflict of interest that could be perceived as prejudicial to the impartiality of the reported research.

\section{Authors Contributions}

RR and AY performed the acquisition of data and wrote the draft. VF and WJ designed the study and evaluated the patient's personal and familial history of cancer. AY and SN performed the bioinformatics analysis. PK helped in the data interpretation of experiments. FC, AS and MB provided the clinicopathological data. All authors reviewed the manuscript for submission.

\section{References}

Alexandrov LB, Nik-Zainal S, Wedge DC, Aparicio SA, Behjati S, Biankin AV, Bignell GR, Bolli N, Borg A, Børresen-Dale AL et al. (2013) Signatures of mutational processes in human cancer. Nature 500:415-421.
Allgäuer M, Budczies J, Christopoulos P, Endris V, Lier A, Rempel E, Volckmar AL, Kirchner M, von Winterfeld M, Leichsenring J et al. (2018) Implementing tumor mutational burden (TMB) analysis in routine diagnostics-a primer for molecular pathologists and clinicians. Transl Lung Cancer Res 7:703715.

Andrianova MA, Chetan GK, Sibin MK, Mckee T, Merkler D, Narasinga RK, Ribaux P, Blouin JL, Makrythanasis P, Seplyarskiy VB et al. (2017) Germline PMS2 and somatic POLE exonuclease mutations cause hypermutability of the leading DNA strand in biallelic mismatch repair deficiency syndrome brain tumors. J Pathol 243:331-341.

Barbari SR and Shcherbakova PV (2017) Replicative DNA polymerase defects in human cancers: Consequences, mechanisms, and implications for therapy. DNA Repair (Amst) 56:16-25.

Barbari SR, Kane DP, Moore EA and Shcherbakova PV (2018) Functional analysis of cancer-associated DNA polymerase $\varepsilon$ variants in Saccharomyces cerevisiae. G3 (Bethesda) 8:1019-1029.

Bellone S, Bignotti E, Lonardi S, Ferrari F, Centritto F, Masserdotti A, Pettinella F, Black J, Menderes G, Altwerger G et al. (2017) Polymerase $\varepsilon$ (POLE) ultra-mutation in uterine tumors correlates with $\mathrm{T}$ lymphocyte infiltration and increased resistance to platinum-based chemotherapy in vitro. Gynecol Oncol 144:146-152.

Billingsley CC, Cohn DE, Mutch DG, Hade EM and Goodfellow PJ (2016) Prognostic significance of POLE exonuclease domain mutations in high grade endometrioid endometrial cancer on survival and recurrence: a sub-analysis. Int J Gynecol Cancer 2:933-938.

Bourdais R, Rousseau B, Pujals A, Boussion H, Joly C, Guillemin A, Baumgaertner I, Neuzillet C and Tournigand C (2017) Polymerase proofreading domain mutations: New opportunities for immunotherapy in hypermutated colorectal cancer beyond MMR deficiency. Crit Rev Oncol Hematol 113:242248.

Briggs S and Tomlinson S (2013) Germline and somatic polymerase $\varepsilon$ and $\delta$ mutations define a new class of hypermutated colorectal and endometrial cancers. J Pathol 230:148-153.

Burgers PM and Kunkel TA (2017) Eukaryotic DNA replication fork. Annu Rev Biochem. 20:417-438.

Büttner R, Longshore JW, López-Ríos F, Merkelbach-Bruse S, Normanno N, Rouleau E and Penault-Llorca F (2019) Implementing TMB measurement in clinical practice: Considerations on assay requirements. ESMO Open 4:e000442.

Endris V, Buchhalter I, Allgäuer M, Rempel E, Lier A, Volckmar AL, Kirchner M, von Winterfeld M, Leichsenring J, Neumann $\mathrm{O}$ et al. (2019) Measurement of tumor mutational burden (TMB) in routine molecular diagnostics: In silico and real-life analysis of three larger gene panels. Int $\mathrm{J}$ Cancer 144:2303-2312.

Forbes SA, Beare D, Boutselakis H, Bamford S, Bindal N, Tate J, Cole CG, Ward S, Dawson E, Ponting L et al. (2017) COSMIC: somatic cancer genetics at high-resolution. Nucleic Acids Res 45:777-783.

Haradhvala NJ, Kim J, Maruvka YE, Polak P, Rosebrock D, Livitz D, Hess JM, Leshchiner I, Kamburov A, Mouw KW et al. (2018) Distinct mutational signatures characterize concurrent loss of polymerase proofreading and mismatch repair. Nat Commun 9:1746.

Heitzer E and Tomlinson I (2014) Replicative DNA polymerase mutations in cancer. Curr Opin Genet Dev 24:107-113. 
Hoeck AV, Tjoonk NH, van Boxtel R and Cuppen E (2019) Portrait of a cancer: Mutational signature analyses for cancer diagnostics. BMC Cancer 19:457-470.

Howitt BE, Shukla SA, Sholl LM, Ritterhouse LL, Watkins JC, Rodig S, Stover E, Strickland KC, D'Andrea AD, Wu CJ et al. (2015) Association of polymerase e-mutated and microsatellite-instable 409 endometrial cancers with neoantigen load, number of tumor-infiltrating lymphocytes, and 410 expression of PD-1 and PD-L1. JAMA Oncol 1:1319-1323.

Imboden S, Nastic D, Ghaderi M, Rydberg F, Rau TT, Mueller MD, Epstein E and Carlson JW (2019) Phenotype of POLEmutated endometrial cancer. PLoS One 14:e0214318.

Levine DA, Getz G, Gabriel SB, Cibulskis K, Lander E, Sivachenko A, Sougnez C, Lawrence M, Kandoth C, Dooling D et al. (2013) Integrated genomic characterization of endometrial carcinoma. Nature 497:67-73.

Liu Y (2019) Immune response characterization of endometrial cancer. Oncotarget 10:982-992.

McConechy MK, Talhouk A, Leung S, Chiu D, Yang W, Senz J, Reha-Krantz LJ, Lee CH, Huntsman DG, Gilks CB et al. (2016) Endometrial carcinomas with POLE exonuclease domain mutations have a favorable prognosis. Clin Cancer Res 22:2865-2873.

Meléndez B, van Campenhout C, Rorive S, Remmelink M, Salmon I and D'Haene N (2018) Methods of measurement for tumor mutational burden in tumor tissue. Transl Lung Cancer Res 7:661-667.

Muzny DM, Bainbridge MN, Chang K, Dinh HH, Drummond JA, Fowler G, Kovar CL, Lewis LR, Morgan MB, Newsham IF et al. (2012) Comprehensive molecular characterization of human colon and rectal cancer. Nature 487:330-337.

Palles C, Cazier JB, Howarth KM, Domingo E, Jones AM, Broderick P, Kemp Z, Spain SL, Guarino E, Salguero I et al. (2013) Germline mutations in the proof-reading domains of
POLE and POLD1 predispose to colorectal adenomas and carcinomas. Nat Genet 45:136-144.

Rayner E, van Gool IC, Palles C, Kearsey SE, Bosse T, Tomlinson I and Church DN (2016) A panoply of errors: polymerase proofreading domain mutations in cancer. Nat Rev Cancer 16:71-81.

Shinbrot E, Henninger EE, Weinhold N, Covington KR, Göksenin AY, Schultz N, Chao H, Doddapaneni H, Muzny DM, Gibbs RA et al. (2014) Exonuclease mutations in DNA polymerase epsilon reveal replication strand specific mutation patterns and human origins of replication. Genome Res 24:1740-1750.

Wong A, Kuick CH, Wong WL, Tham JM, Mansor S, Loh E, Jain S, Vikas NN, Tan SH, Chan SH et al. (2016) Mutation spectrum of POLE and POLD1 mutations in South East Asian women presenting with grade 3 endometrioid endometrial carcinomas. Gynecol Oncol 141:113-20.

Zhang J, Baran J, Cros A, Guberman JM, Haider S, Hsu J, Liang Y, Rivkin E, Wang J, Whitty B et al. (2011) International Cancer Genome Consortium Data Portal: A one-stop shop for cancer genomics data. Database (Oxford) 2011:bar026.

\section{Supplementary material}

The following online material is available for this article: Mat-Met S1 - Detailed description of all material and methods used in this study.

Table S1 - Detailed list of the 63 genes used for targeted sequencing.

Associate Editor: Daisy Maria Fávero Salvadori

License information: This is an open-access article distributed under the terms of the Creative Commons Attribution License (type CC-BY), which permits unrestricted use, distribution and reproduction in any medium, provided the original article is properly cited. 